\title{
A Kind of Multi-Channel Priority Control Device for Greenhouse Roller Shutter Machine
}

\author{
He Gong ${ }^{1, ~ a ~}$, Bin Liu ${ }^{1, b}$, Hongyan Ju ${ }^{1, c}$ and $\mathrm{Ji} \mathrm{Li}^{1, \mathrm{~d}}$ \\ ${ }^{1}$ Information \& Technology College, Jilin Agriculture University, Changchun 130118, China \\ agonghe0001@163.com, 'bliubin062690@163.com, ' didiaoxiaoju@163.com, 'liji1996@163.com
}

Keywords: Greenhouse, Roller shutter machine, Multi-channel priority.

\begin{abstract}
The greenhouse roller shutter machines available mostly are pointing controlled through motor, existing the security problems like easy falling of heat-preserving curtain rolled up. This paper presents a kind of multi-channel priority control device for greenhouse roller shutter machine, achiving the multi-channel control which contains limit control, manual control, remote control and program control for roller shutter machine. The device could improved using safety while the system was controlled more accurately and intelligently.
\end{abstract}

\section{Introduction}

The greenhouse, which is instrumental in breeding the anti-season vegetables, shortening the growth cycle of crops and improving the crops survival rate, has important significance in the development of agricultural production. There are more and more requirements for the regulation of greenhouse environment parameters with the agricultural modernization rapidly developing and continuous expansion of the greenhouse.

The roller shutter machine of greenhouse is an agricultural machinery equipment, which is designed to control the heat-preserving curtain lifting [1]. According to the researches, the incomes per mu of breeding melons, vegetables and edible fungus were more than 6,000 yuan and even up to 10,000 yuan. Particularly the great profits of anti-seasonal vegetables and fruits were the key factors that farmer's enthusiasm on greenhouse crops production [2]. Plastic greenhouse production bases were built all over the country for vegetables breeding and fresh fruit production, and the scale was increasing constantly. In the greenhouse operation, rolling up and laying the heat-preserving curtain was one of the most time-consuming work, especially at the early morning and evening in the winter. The work in terrible conditions was badly limited the yield profit and development space of greenhouse due to the strong labor intensity and low efficiency. The appearance of roller shutter machine met the farmer's demand for greenhouse crops production. The machine not only can solve the problem existing in manual rolling up the heat-preserving curtain, but it's quick operation also makes the illumination time prolonged and crop quality improved which give farmers more energy managing crops easy. In addition, the application of roller shutter machine has a better protection for grass curtain and cotton curtain, and prolongs the service life of curtains thus cutting production costs.

\section{The Conventional Roller Shutter Machine}

The roller shutter machine could be divided into front machine and post machine according to the installation method, and also could be divided into electric machine and artificial machine according to the different power source. The machine most frequently using power was $220 \mathrm{~V}$ or $380 \mathrm{~V}$ alternating current [3]. The roller shutter machines available mostly were pointing controlled through motor, and existed the security problems of falling easily of heat-preserving curtain rolled up due to the imperfect control system at present. Electrical motor turned while the finger pressing the button, otherwise the motor would stop and the position of heat-preserving curtain was fully determined by manual control. The phenomenon of rolled heat-preserving curtain falling from the top of greenhouse was often appeared because of miscarriage of justice and the power couldn't be cut off in time, which 
contributed to the broke of support arm, rocker arm and reel bended. The repair of roller shutter machine damaged was expensive and time-consuming, even causing danger of injury further.

To solve the problem above, this paper designs a kind of multi-channel priority control device for greenhouse roller shutter machine.

\section{Overall Framework}

The multi-channel priority control device for greenhouse roller shutter machine consists of JN5148 wireless micro controller unit, wireless receiving module, decoding circuit, key de-twitter circuit, multi-channel priority logic circuit, photoelectric isolation circuit, relay control circuit, AC contactor, three phase motor control circuit, limited switch, indicator circuit and power management unit. The whole system's block diagram is shown as Fig. 1.

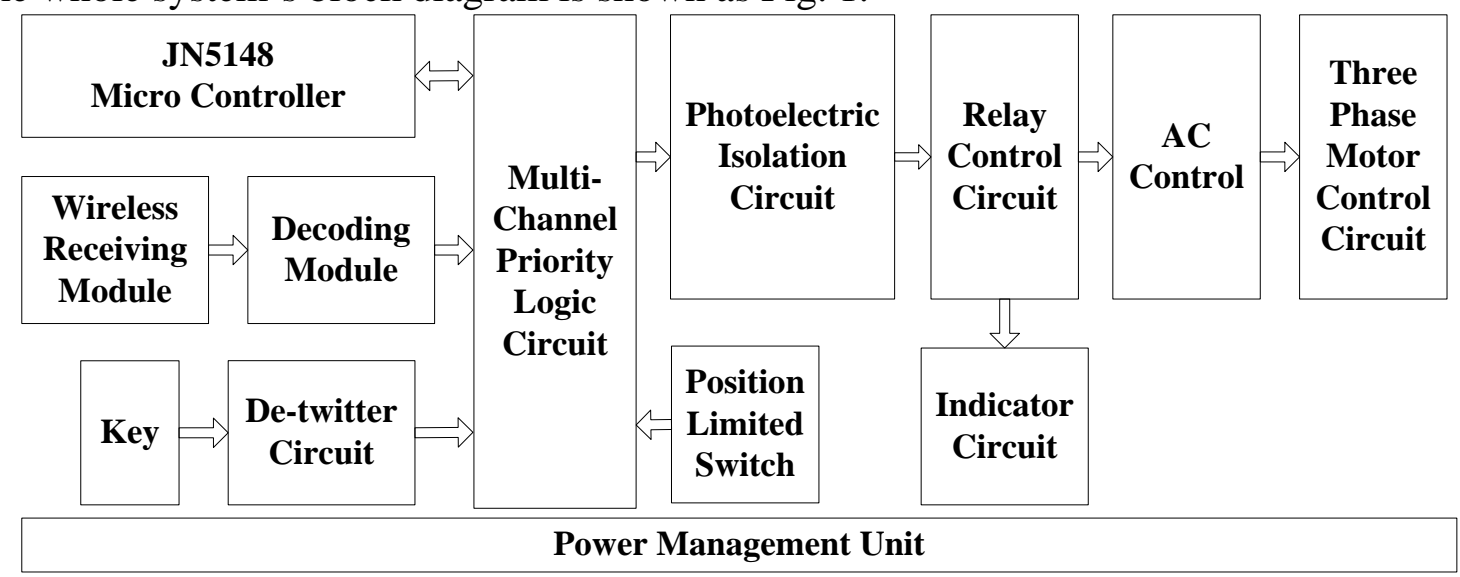

Fig. 1 Block Diagram of Device

\section{Priority of Control Methods.}

The control methods of device for greenhouse roller shutter machine divides into program control, wireless control, manual button control and position limited control. Using JN5148 wireless micro controller achieves the program control, and the method of remote control is applying RXB8 ultra high frequency wireless transmitting and receiving module. Using the key de-twitter circuit realizes manual button control. The aim of limited switches at the highest and lowest of running trajectory is to detect the heat-preserving curtain position and prevent the damage causing by negligent operation at the highest. The priority order of different control methods above is shown as Fig. 2.

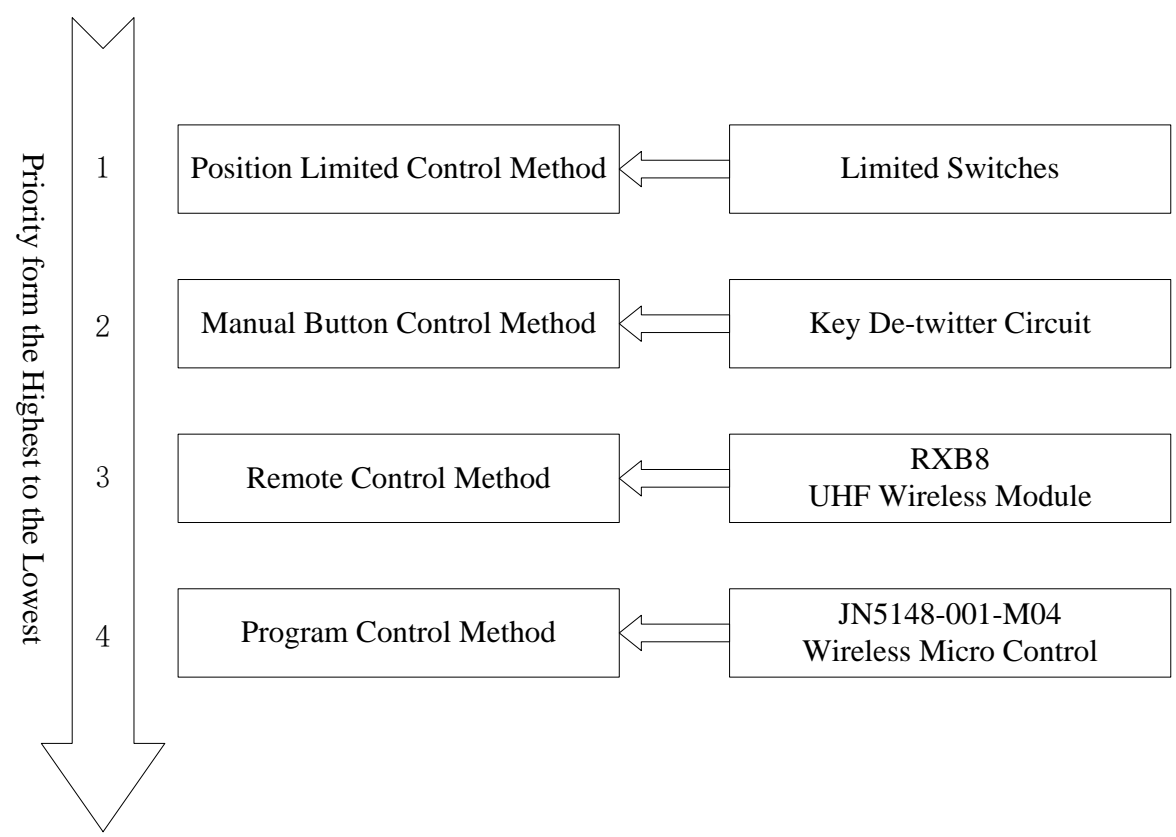

Fig. 2 Block Diagram of Priority Order 


\section{Realization of Program Control.}

The system applies JN5148-001-M04 which is NXP Company launched as main processor to program control. The device features an enhanced 32-bit RISC processor and includes a $2.4 \mathrm{GHz}$ IEEE 802.15.4 compliant transceiver, $128 \mathrm{kB}$ of ROM, $128 \mathrm{kB}$ of RAM, and a rich mix of analogue and digital peripherals, including $\mathrm{I}^{2} \mathrm{C}$, SPI, ADC interface and so on. Four-wire $\mathrm{I}^{2} \mathrm{~S}$ audio interface can connect with the main trend decodes directly [4]. The highest transmission distance can achieve $1000 \mathrm{~m}$. [5]. In software development, Jennic provides a wealth routine and code, making software relatively easy to develop.

\section{Realization of Wireless Control.}

RXB8 is a kind of ultra high frequency wireless data transmitting and receiving module. Receiving distance of the module with sensitivity up to $-114 \mathrm{dBm}$ is more than twice the normal receiving modules. It's work frequencies is $315 \mathrm{MHz}$ and $433.92 \mathrm{MHz}$, and the module has low power design with 9.6mA current waste at 5V power. The RXB8 schematic circuit diagram is shown as Fig. 3 .

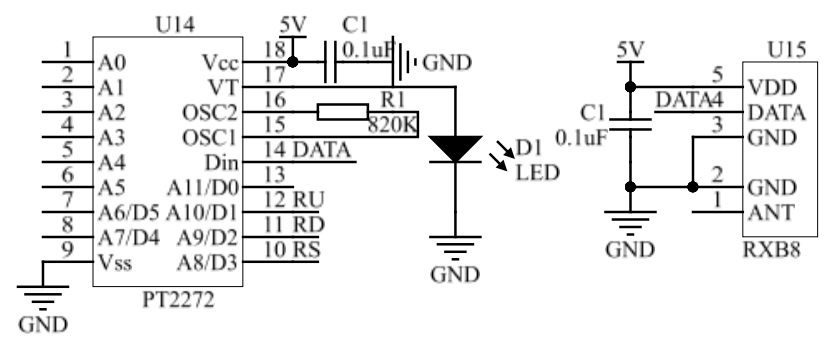

\section{Realization of Multi-Channel Priority Control.}

Fig. 3 The RXB8 Schematic Circuit Diagram

The device realizes multi-channel priority control for greenhouse roller shutter machine through the priority logic circuit. Once the signals from these different control methods arrive together, the priority logic circuit would determine the highest priority of them being the output signal for controlling the machine. When the signals which are same priority like up signal and down signal arrives, the priority logic circuit would determine the output being stop control signal. The priority logic schematic circuit diagram is shown as Fig. 4.
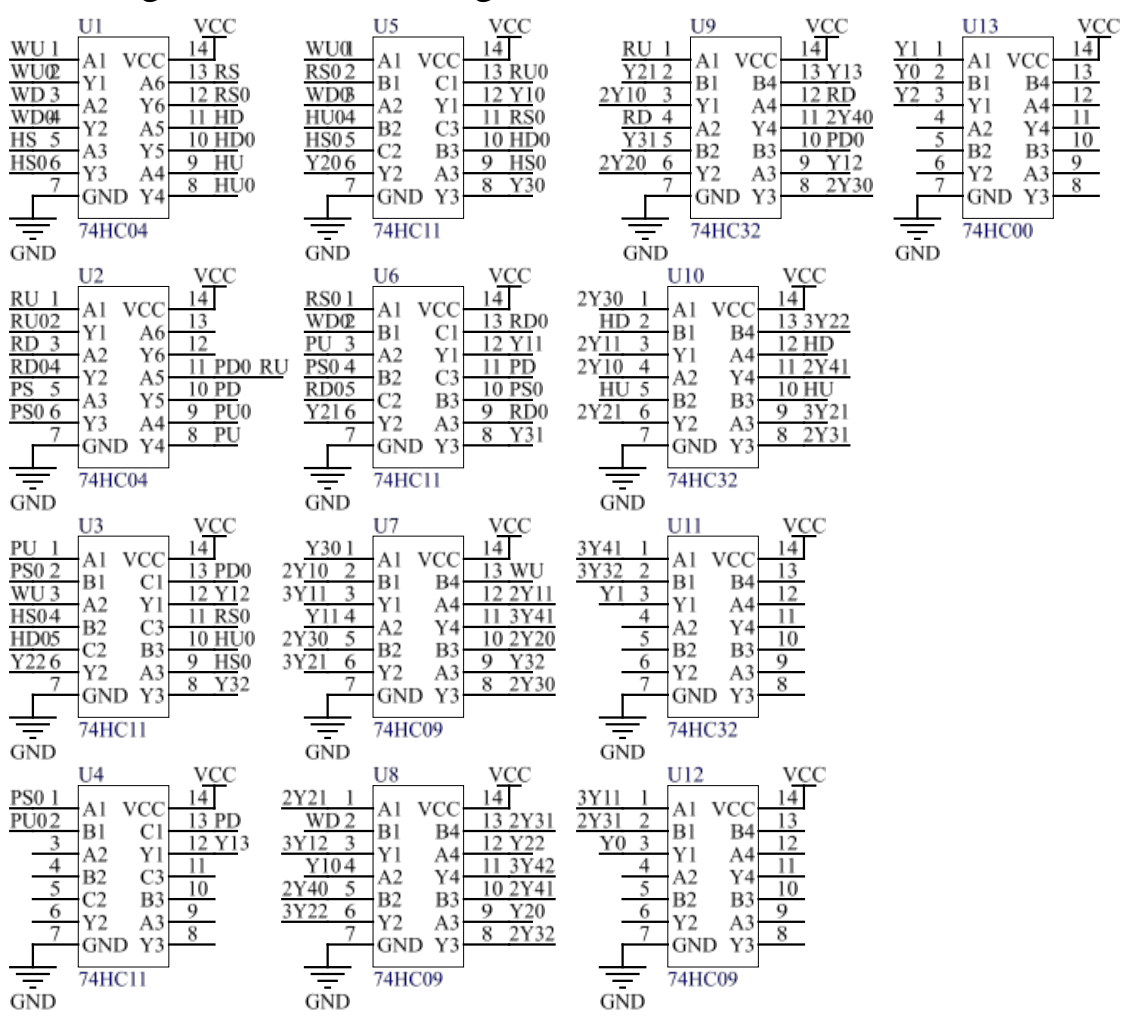

Fig. 4 The Priority Logic Schematic Circuit Diagram 


\section{Conclusions}

The device presented by this paper could realize multi-channel priority control for greenhouse roller shutter machine, for instance, when the top limit switch signal is high level, manual down control signal is low level, and other signals are any level, the output signals are as follows: motor run forward signal and reversal signal are low level while motor stop signal is high level. When the top limit switch signal is high level, manual control signals containing up, down and stop signals are low level, remote down control is high as well as remote up and stop signals are low level, and other signals are any level, the output signals are as follows: motor run forward signal and motor stop signal are low level while motor run reversal signal is high level. When the limit switches' signals are low level, manual up control signal is high level, and rest manual control signals and remote control signals are low level, program down control signal is high level and rest program control signals are low level, the output signals are as follows: motor run reversal signal and motor stop signal are low level while motor run forward signal is high level. When limit switches' signals are low level, manual control signals and remote control signals are low level, program down control signal is high and the remaining program control signals are low level, then the output signals are as follows: motor run forward signal and motor stop signal are low level and motor run reversal signal is high. When limit switches' signals are low level, manual control signals and remote control signals are low level, program up and down control signals are high level while program stop control signal is low level, then the output signals are as follows: motor run forward signal and motor run reversal signal are low level while motor stop is high level. The security of roller shutter machine is greatly improved due to the application of device this paper presented, meanwhile, the whole system runs more rapidly and accurately.

\section{Acknowledgments}

This work was financially supported by Changchun Science and Technology Bureau project(14NK029), Jilin Education Department projects([2015] No.175 and [2015] No.184) and Jilin Science and Technology Department project(20160623016TC).

\section{References}

[1] G. Liu: Agricultural Science \& Technology and Equipment, (2012), No.11, p.29-30+34

[2] Z.J. Yao, C.T. Li, J.H. Li, Y.S. Li: Agro-Environment \& Development, (2011), No.05, p.102-107

[3] J.Z. Zhang: Contemporary Farm Machinery, (2016), No.01, p.17-18

[4] Information on http://www.jennic.com

[5] P. He: Research of Building Structure Monitoring System Based on Wireless Sensor Network (MS., Jiangnan University, 2012), p.46. 